\title{
Long noncoding RNA LINC01296 promotes tumor growth and progression by sponging miR-5095 in human cholangiocarcinoma
}

\author{
DAWEI ZHANG ${ }^{1 *}$, HAIYAN LI ${ }^{2 *}$, JUPING XIE ${ }^{1}$, DECAN JIANG ${ }^{1}$, LIANGQI CAO ${ }^{1}$, \\ XUEWEI YANG ${ }^{1}$, PING XUE ${ }^{1}$ and XIAOFENG JIANG ${ }^{1}$ \\ ${ }^{1}$ Department of Hepatobiliary Surgery, The Second Affiliated Hospital of Guangzhou Medical University, \\ Guangzhou, Guangdong 510260; ${ }^{2}$ Department of Breast and Thyroid Surgery, \\ The Sixth Affiliated Hospital of Sun Yat-sen University, Guangzhou, Guangdong 510655, P.R. China
}

Received December 23, 2017; Accepted March 12, 2018

DOI: $10.3892 /$ ijo.2018.4362

\begin{abstract}
The aim of the present study was to elucidate whether, and how, long intergenic non-protein coding RNA 1296 (LINC01296) is involved in the modulation of human cholangiocarcinoma (CCA) development and progression. Microarray data analysis and reverse transcription-quantitative polymerase chain reaction analysis demonstrated that LINC01296 was significantly upregulated in human CCA compared with nontumor tissues. Furthermore, the expression of LINC01296 in human CCA was positively associated with tumor severity and clinical stage. Knockdown of LINC01296 dramatically suppressed the viability, migration and invasion of RBE and CCLP1 cells, and promoted cell apoptosis in vitro. Furthermore, LINC01296 knockdown inhibited tumor growth in a xenograft model. Mechanistically, LINC01296 was demonstrated to sponge microRNA-5095 (miR-5095), which targets MYCN proto-oncogene bHLH transcription factor (MYCN) mRNA in human CCA. By inhibition of miR-5095, LINC01296 overexpression upregulated the expression of MYCN and promoted cell viability, migration and invasion in CCA cells. The results reveal that the axis of LINC01296/ miR-5095/MYCN may be a mechanism to regulate CCA development and progression.
\end{abstract}

\section{Introduction}

Cholangiocarcinoma (CCA) is a highly invasive and metastatic cancer with diagnostic difficulties and high mortality (1). In

Correspondence to: Professor Xiaofeng Jiang, Department of Hepatobiliary Surgery, The Second Affiliated Hospital of Guangzhou Medical University, 250 East Changgang Road, Guangzhou, Guangdong 510260, P.R. China

E-mail: jiangxiaofeng008@163.com

*Contributed equally

Key words: long intergenic non-protein coding RNA 1296, proliferation, microRNA-5095, MYCN proto-oncogene bHLH transcription factor, cholangiocarcinoma recent years, the incidence and mortality of CCA in the world are increasing rapidly, according to epidemiological studies $(1,2)$. However, the pathogenesis of CCA still remains unclear (3). The male/female ratio in incidence of CCA is $1.4: 1$, and it often occurs in men aged between 50 and 70 years old $(4,5)$. Hepatitis $\mathrm{B}$ and hepatitis $\mathrm{C}$ infection is potentially associated with the occurrence of CCA (6). Surgery is the main effective treatment for CCA; however, the postoperative recurrence rate is high, and the 5-year survival rate is only $5 \%(7,8)$. For the majority of patients with terminal CCA, surgical treatment and liver transplantation are not the ideal treatment. Conventional radiotherapy and chemotherapy exhibit limited therapeutic effect on patients with CCA. Therefore, it is imperative to seek effective treatments and identify sensitive biomarkers for early diagnosis of CCA. Molecular targeted therapy possesses wide application potentially with highly targeted and fewer adverse reactions, which may control cancer cell proliferation, and prevent or delay recurrence metastasis (9). Therefore, there is an urgent need to understand of the molecular mechanisms underlying carcinogenesis and progression of CCA, which contribute to finding novel diagnosis markers and novel therapeutic targets.

In recent years, noncoding RNAs, including long noncoding RNAs (lncRNAs) and microRNAs (miRNAs/miRs), have been demonstrated to have important functions in various biological processes, particularly in cancers (10-15). The research of oncogenes and noncoding RNAs has been useful for further investigation of the pathogenesis of CCA and novel molecular targeted therapy $(16,17)$. Recent evidences demonstrated that lncRNAs can regulate tumor development and progression by acting as competing endogenous RNAs in various cancers. For instance, the long noncoding RNA taurine upregulated 1 acts as a competing endogenous RNA to sponge miR-132 in hepatocellular carcinoma (18). Long non-coding RNA metastasis associated lung adenocarcinoma transcript 1 (MALAT1) sponges miR-144-3p to promote metastasis and proliferation in osteosarcoma cells (19). miRNAs are a group of small (19-25 nucleotides) endogenous non-coding RNA molecules, which act as gene regulators by binding to partially complementary target sites in mRNA 3' untranslated regions (3'UTRs) that results in degradation of the target mRNAs or translational repression of the encoded proteins (20). Wang et al (21) 
reported that downregulation of miR-138 enhanced the proliferation, migration and invasion of CCA cells through the upregulation of RhoC/phospho-ERK/matrix metalloproteinase-2/9. However, the roles of noncoding RNAs in CCA still remain largely unknown.

In this study, the expression patterns of long intergenic non-protein coding RNA 1296 (LINC01296) and its biological functions in CCA were investigated. The further investigations were conducted to determine the molecular mechanism by which LINC01296 regulates CCA development. Finally, it was demonstrated that LINC0129 interacted with miR-5095 to enhance the expression of MYCN proto-oncogene bHLH transcription factor (MYCN) and promoted CCA development and progression.

\section{Materials and methods}

Patient and tissue samples. Paired CCA tissues $(\mathrm{n}=57)$ and matched peritumor samples were obtained from a tissue bank of samples collected from patients that underwent surgical treatment between January 2010 and December 2016 at the Second Affiliated Hospital of Guangzhou Medical University (Guangzhou, China) (Table I). Tissue samples were snap frozen in liquid nitrogen immediately following surgical resection and stored at $-80^{\circ} \mathrm{C}$. Written informed consent was obtained from all patients. This study did not include patients that had received radiotherapy and/or immunotherapy prior to or following surgical treatment. The study protocol conformed to the ethical guidelines of the 1975 Declaration of Helsinki, and was approved by The Second Affiliated Hospital of Guangzhou Medical University.

Fluorescence in situ hybridization (FISH). A FISH kit was purchased from Guangzhou Boye Biological Technology Co., Ltd. (Guangzhou, China) and fluorescein isothiocyanate (FITC)-conjugated LINC01296 DNA probe (5'-TATGGG AAGGGGACTGTCTG-3') was used for RNA-FISH as previously described (22). In brief, CCA tissues were fixed in $4 \%$ paraformaldehyde for $48 \mathrm{~h}$ at $37^{\circ} \mathrm{C}$. Paraffin sections $(7 \mu \mathrm{m}$ in thickness) were processed by dewaxing, protein removal and pre-hybridization, and then hybridized with probe. DAPI staining was performed and observed with a FV1000 confocal laser microscope (Olympus Corporation, Tokyo, Japan).

In situ hybridization (ISH). ISH was performed as previously described (23). In brief, samples were fixed and embedded in paraffin. Then, sample sections were incubated in graded alcohols and incubated in $3 \%$ hydrogen peroxide $\left(\mathrm{H}_{2} \mathrm{O}_{2}\right)$ for $30 \mathrm{~min}$. Biotin-conjugated probes and streptavidin-horseradish peroxidase conjugate were used for ISH. The samples were finally stained with hematoxylin and observed with a light microscope (Nikon Corporation, Tokyo, Japan). The LINC01296 DNA probe sequence was 5'-CTCCCTCAAATCAGGATGGG-3'

Cell culture and transfection. CCA cell lines, including HIBEC noncancerous cholangiocyte cell line, and RBE, CCLP1, HuCCT1 and HCCC-9810 cholangiocarcinoma cell lines, were purchased from the Chinese Academy of Sciences Cell Bank (Shanghai, China). All cells were cultured in RPMI-1640 (Gibco; Thermo Fisher Scientific, Inc., Waltham, MA, USA) supplemented with $10 \%$ fetal bovine serum (FBS; Invitrogen;
Table I. Association between clinicopathological features and LINC01296 expression in 57 patients with cholangiocarcinoma.

\begin{tabular}{lccc}
\hline & \multicolumn{2}{c}{ LINC01296 expression } & \\
\cline { 2 - 3 } Feature & Low & High & P-value \\
\hline All cases & 35 & 22 & \\
Age (year) & & & 0.553 \\
$\quad<60$ & 34 & 20 & \\
$\geq 60$ & 1 & 2 & \\
Sex & & & 0.789 \\
Male & 19 & 13 & \\
Female & 16 & 9 & \\
Size (cm) & & & 0.003 \\
$<3$ & 21 & 4 & \\
$\geq 3$ & 14 & 18 & \\
Lymph node metastasis & & & \\
No & 22 & 7 & \\
Yes & 13 & 15 & \\
TNM & & & \\
I/II & & & \\
III/IV & 9 & 13 & \\
\hline
\end{tabular}

The mean expression level was used as the cutoff. For analysis of association between LINC01296 levels and clinical features, Pearson's $\chi^{2}$ tests were used. LINC01296, long intergenic non-protein coding RNA 1296; TNM, tumor node metastasis stage.

Thermo Fisher Scientific, Inc.) in humidified $5 \% \mathrm{CO}_{2}$ at $37^{\circ} \mathrm{C}$. miR-5095 mimics (5'-UUACAGGCGUGAACCACCGCG-3'), inhibitor (5'-CGCGGTGGTTCACGCCTGTAA-3'), short hairpin RNA(shRNA) which targetedLINC0129(shLINC0129, 5'-GGUUCAUCUGUGUUGCUCU-3') and relative controls (5'-AATTCTCCGAACGTGTCACGT-3') were obtained from GenePharma Co., Ltd. (Shanghai, China). The transfection was conducted by using Lipofectamine ${ }^{\circledR} 2000$ (Invitrogen; Thermo Fisher Scientific, Inc.) in $5 \times 10^{6}$ cells at a final concentration of $50 \mathrm{nM}$. Subsequent assays were performed at $48 \mathrm{~h}$ after transfection. To overexpress LINC01296, CCA cells were transfected with pCDNA3-LINC01296 using Lipofectamine ${ }^{\circledR} 2000$. After transfection for $24 \mathrm{~h}$, expression of LINC01296 was validated by reverse transcription-quantitative polymerase chain reaction (RT-qPCR).

$R T-q P C R$. Total RNA was extracted from $1 \times 10^{7} \mathrm{CCA}$ and noncancerous cholangiocyte cells using the TRIzol reagent (Thermo Fisher Scientific, Inc.) according to the manufacturer's instructions. For miRNA analysis, RT-qPCR was performed using the TaqMan MicroRNA Reverse Transcription kit, TaqMan Universal PCR Master Mix (Applied Biosystems; Thermo Fisher Scientific, Inc.) according to the manufacturer's instructions with corresponding primers: Universal miRNA RT-qPCR primer, 5'-AACGAGACGACGACAGAC-3'; 5'-GCAAATTCGTGAAG CGTTCCATA-3' for U6; and 5'-TACAGG CGTGAACCACC-3' for miR-5095. For mRNA analysis, RT-qPCR was performed 
using the TaqMan High-Capacity cDNA Reverse Transcription Kit, TaqMan Fast PCR Master Mix (Applied Biosystems; Thermo Fisher Scientific, Inc.) according to the manufacturer's instructions with corresponding primers. GAPDH was used as an internal control to normalize MYCN. The RT-qPCR protocol included an initial denaturation step $\left(95^{\circ} \mathrm{C}\right.$ for $\left.5 \mathrm{~min}\right)$ and 40 cycles of denaturation $\left(95^{\circ} \mathrm{C}\right.$ for $\left.10 \mathrm{sec}\right)$, annealing $\left(60^{\circ} \mathrm{C}\right.$ for $20 \mathrm{sec})$ and extension $\left(72^{\circ} \mathrm{C}\right.$ for $\left.10 \mathrm{sec}\right)$. The relative expression levels were calculated using $2^{-\Delta \Delta \mathrm{Cq}}$ method as previously described (24). The primer sequences were as follows: LINC01296 (forward, 5'-GAGAAGCAGTGGTGGGTTCC-3' and reverse, 5'-GAGCAACACAGATGAACCGC-3'), MYCN (forward, 5'-ACTGTAGCCATCCGAGGACA-3' and reverse, 5'-CAAGCCCTGCTCCTTACCTC-3') and GAPDH (forward, 5'-TCCTCTGACTTCAACAGCGACAC-3' and reverse, 5'-CAC CCTGTTGCTGTAGCCAAATTC-3').

Cell proliferation assay. Cells were seeded at 5,000 cells per well in 96-well plates at $24 \mathrm{~h}$ after transfection. Cell proliferation was measured using the Cell Counting Kit-8 (CCK8; Dojindo Molecular Technologies, Inc., Kumamoto, Japan) at 24, 48 and $72 \mathrm{~h}$ after the cells were seeded. Absorbance was determined at $450 \mathrm{~nm}$ using a microplate spectrophotometer (Thermo Fisher Scientific, Inc.).

Cell cycle analysis. Cells were harvested at $48 \mathrm{~h}$ after transfection. The cells were washed with PBS and fixed in ethanol at $-20^{\circ} \mathrm{C}$. The cells were then washed with PBS, rehydrated and resuspended in propidium iodide (PI; $10 \mu \mathrm{l}$ )-RNase A solution (Sigma-Aldrich; Merck KGaA) at $37^{\circ} \mathrm{C}$ for $30 \mathrm{~min}$. The stained cells $\left(1 \times 10^{5}\right)$ were then analyzed for DNA content with a flow cytometer (BD Biosciences, Franklin Lakes, NJ, USA). The results were analyzed with FlowJo 7.6.1 software (FlowJo LLC, Ashland, OR, USA).

Cell apoptosis analysis. Cells were harvested, washed with ice-cold PBS, and stained with Annexin V-FITC apoptosis detection kits (Nanjing KeyGen Biotech Co., Ltd., Nanjing, China). Cell apoptosis was analyzed using a flow cytometer (BD Biosciences). The results were analyzed with FlowJo 7.6.1 software.

Cell migration and invasion assay. Cell migration was evaluated using a wound-healing assay. In brief, at $48 \mathrm{~h}$ after transfection, cells were cultured in 6 -well plates $\left(5 \times 10^{4}\right.$ cells per well). At $90-95 \%$ confluence, the monolayer of cells was scratched with using a sterile plastic micropipette tip, and then cell were cultured under standard conditions for $24 \mathrm{~h}$. Following several washes, recovery of the wound was observed and imaged using an X71 inverted microscope (Olympus Corporation).

A Transwell invasion assay was performed to determine cell invasion. Transfected cells $\left(1 \times 10^{5}\right)$ were seeded into the upper chamber of Matrigel-coated inserts with free-serum medium. Medium with $10 \%$ FBS was added to the lower chamber as chemoattractant. The cells were allowed to invade for $48 \mathrm{~h}$ at $37^{\circ} \mathrm{C}$ with $5 \% \mathrm{CO}_{2}$. Then cells invaded to the lower surface of filter were fixed in $70 \%$ ethanol for $30 \mathrm{~min}$ and stained with $0.1 \%$ crystal violet for $10 \mathrm{~min}$ at $25^{\circ} \mathrm{C}$. The number of cells that migrated to the lower side was counted in five randomly selected fields under an X71 inverted microscope (Olympus Corporation).

Luciferase activity assay. Wild-type LINC01296 or MYCN 3'UTR sequences were amplified from a human cDNA library. Mutations of the miR-5095 binding site were introduced by sitedirected mutagenesis using a fast mutation kit (New England BioLabs, Inc., Ipswich, MA, USA). The PCR fragment was cloned into psiCHECK-2 vector (Addgene, Inc., Cambridge, MA, USA) downstream of the firefly luciferase coding region within XhoI and NotI (Takara Bio, Inc., Otsu, Japan). psiCHECK2 -control was used as internal control. The psiCHECK-2 reporter plasmids $\left(1 \mu \mathrm{g}\right.$ per well) were tranfected into $1 \times 10^{6}$ CCA cells using Lipofectamine ${ }^{\circledR} 2000$ (Invitrogen; Thermo Fisher Scientific, Inc.). At $48 \mathrm{~h}$ after transfection, the luciferase activity was measured as previously described (25). The primer sequences for LINC01296 or MYCN cloning were as follows: LINC01296 (forward, 5'-CCCTGCAGCAGCAGCGGCGTG-3' and reverse, 5'-ACACTCACACACACTCCCACC-3') and MYCN (forward, 5'-ACGCTTCTCAAAACTGGACAG-3' and reverse, 5'-AGCTATTTATTTTCATAAACATG-3').

Western blot analysis. Total protein lysates were resolved by $10 \%$ SDS-PAGE and transferred to polyvinyl difluoride membranes (EMD Millipore, Billerica, MA, USA). Following blocking in Tris-buffered saline containing $0.1 \%$ Tween-20 (TBS-T) with 5\% nonfat dry milk for $30 \mathrm{~min}$ at $37^{\circ} \mathrm{C}$, membranes were washed four times in TBS-T and incubated with primary antibodies overnight at $4^{\circ} \mathrm{C}$. Primary antibodies were all obtained from Abcam (Cambridge, MA, USA) and used at the following dilutions: Anti-caspase-3 (1:300; cat. no. 3550914; BD Biosciences), Twist1 (1:500; cat. no. 46702), anti-GAPDH (1:1,000; cat. no. 5174), anti-Cyclin D1 (1:1,000; cat. no. 2978), anti-matrix metalloproteinase-2 (1:1,000; cat. no. 87809) and anti-MYCN $(1: 2,000$; cat. no. 84406) (all from CST Biological Reagents Co., Ltd.). Following extensive washing, membranes were incubated with horseradish peroxidase-linked goat polyclonal anti-rabbit IgG secondary antibody (cat. no. 7074; CST Biological Reagents Co., Ltd.) at a dilution of 1:2,000 for $1 \mathrm{~h}$ at room temperature. Immunoreactivity was detected by enhanced chemiluminescence (Pierce; Thermo Fisher Scientific, Inc.) and visualized using a ChemiDoc XRS imaging system and analysis software (Bio-Rad Laboratories, Inc., Hercules, CA, USA). GAPDH served as the loading control.

In vivo xenograft experiments. Male BALB/c nude mice (6-week-old; $n=6$ ) were purchased from Beijing HFK Bioscience Co. Ltd. (Beijing, China) and maintained under pathogen-free conditions with approval by the Committee of the Second Affiliated Hospital of Guangzhou Medical University. For tumor propagation analysis, $1 \times 10^{7} \mathrm{RBE}$ tumor cells were subcutaneously injected into BALB/c nude mice. Tumor volume was calculated using the formula: volume $=\pi a b^{2} / 6$ (a, tumor length; $b$, tumor width) at indicative time points. Tumor weight was measured at week 4 post-injection. Animal experiments were performed in accordance with relevant guidelines and regulations of the Animal Care and Use Committees at the Second Affiliated Hospital of Guangzhou Medical University, and a signed document 


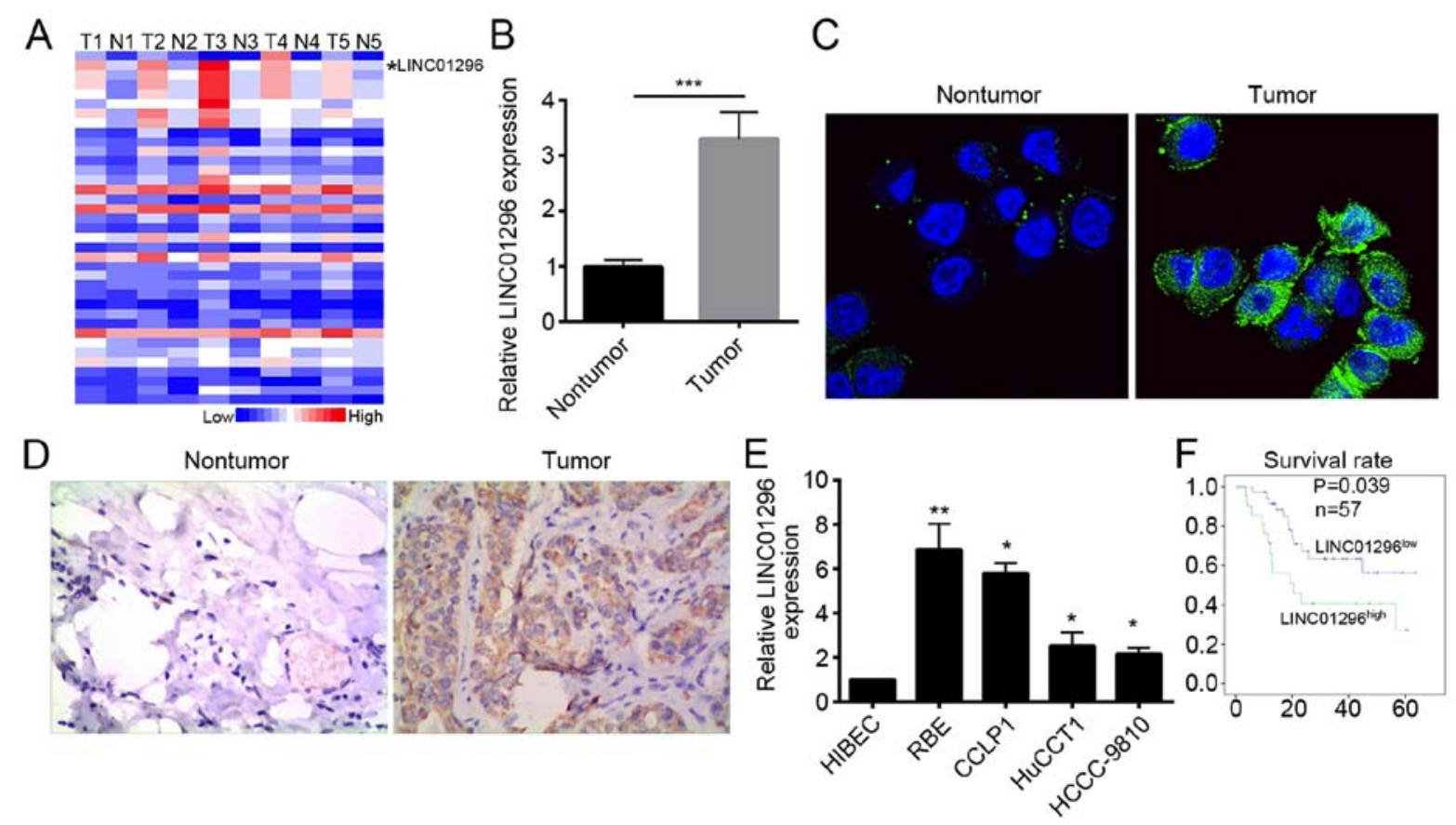

Figure 1. LINC01296 is upregulated in human CCA. (A) LINC01296 upregulation in human CCA samples by bioinformatics analysis according to the dataset (GSE61850). (B) Analysis of LINC01296 expression levels in 57 paired human CCA samples and nontumor tissue by RT-qPCR, ${ }^{* * *} \mathrm{P}<0.001$. (C) Fluorescent in situ hybridization and (D) in situ hybridization probing for LINC01296 in CCA and nontumor samples. (E) Expression levels of LINC01296 in CCA cell lines (RBE, CCLP1, HuCCT1 and HCCC-9810) and noncancerous cholangiocyte cell line (HIBEC) were determined by RT-qPCR. ${ }^{*} \mathrm{P}<0.05$ and ${ }^{* *} \mathrm{P}<0.01$ vs. HIBEC. (F) Survival rates of patients with CCA with high and low LINC01296 by Kaplan-Meier survival analysis. Data are presented as the mean \pm standard deviation. CCA, cholangiocarcinoma; RT-qPCR, reverse transcription-quantitative polymerase chain reaction; LINC01296, long intergenic non-protein coding RNA 1296.

issued by the Animal Care and Use Committees that granted approval was obtained.

Cohort analysis. Online-available data set (GSE61850) was downloaded from NCBI (ncbi.nlm.nih.gov/gds/?term¹/4). $\mathrm{R}$ language (r-project.org) and Bioconductor (bioconductor.org/) were used for background correction, normalization, expression calculation and annotation. The gene expression profiles were evaluated for further analyses using Excel (Microsoft Corporation, Redmond, WA, USA), SPSS 19.0 statistical software (version 19.0; IBM Corp., Armonk, NY, USA) or GraphPad Prism 5 software (GraphPad Software, Inc., La Jolla, CA, USA).

Colony formation assays. Colony formation assays were used to assess clonogenic ability of transfected cells. CCA cells ( $2 \times 10^{3} /$ well) were trypsinized in a single-cell suspension and seeded in 6-well dishes. Subsequently, the cells were maintained in RPMI-1640 supplemented with $10 \%$ fetal bovine serum for about 2 weeks. The visible colonies were fixed in $4 \%$ paraformaldehyde for $4 \mathrm{~h}$ at $37^{\circ} \mathrm{C}$ and stained with $0.5 \%$ crystal violet for $2 \mathrm{~h}$ at $37^{\circ} \mathrm{C}$ (Beyotime Institute of Biotechnology, Haimen, China). Colony numbers were counted under a light microscope (Olympus Corporation).

Statistical analysis. Data from at least three independent experiments are expressed as the mean \pm standard deviation. Statistical analysis was performed using IBM SPSS 19.0 statistical software. Statistical analysis was performed using Student's t-test or ANOVA. Pearson's $\chi^{2}$ tests were used for analysis of the correlation between clinicopathological features and LINC01296 expression in CCA patients. KaplanMeier survival analysis was used for analysis of survival rate and P-value was calculated by the log-rank test. Spearman's correlation analysis was used to determine the correlations between the levels of LINC01296 and miR-5095 in CCA tissues. $\mathrm{P}<0.05$ was considered to indicate a statistically significant difference.

\section{Results}

LINC01296 is upregulated in human CCA. To explore important lncRNAs in human CCA, an online dataset (GSE61850) was analyzed and a functionally undefined lncRNA LINC01296 that was greatly upregulated in human CCA tissues was identified (Fig. 1A). To validate this analysis, the expression levels of LINC01296 were measured in 57 pairs of human CCA samples by RT-qPCR, and LINC01296 expression was significantly upregulated in CCA tissues compared with nontumor tissues (Fig. 1B). Additionally, FISH and ISH assays were performed with pairs of CCA samples, which demonstrated that the expression of LINC01296 was higher in tumor tissues than nontumor tissues (Fig. 1C and D). Furthermore, RT-qPCR also showed that the expression of LINC01296 was higher in CCA cell lines (RBE, CCLP1, HuCCT1 and HCCC-9810) compared with HIBEC noncancerous cholangiocyte cell line (Fig. 1E). To further investigate the clinicopathological significance of LINC01296 level in patients with CCA, The 57 patients were divided into 2 subgroups based on the mean value: Low LINC01296 group (35 cases) and a high LINC01296 group (22 cases). As presented in Table I, LINC01296 levels in CCA tissues were 

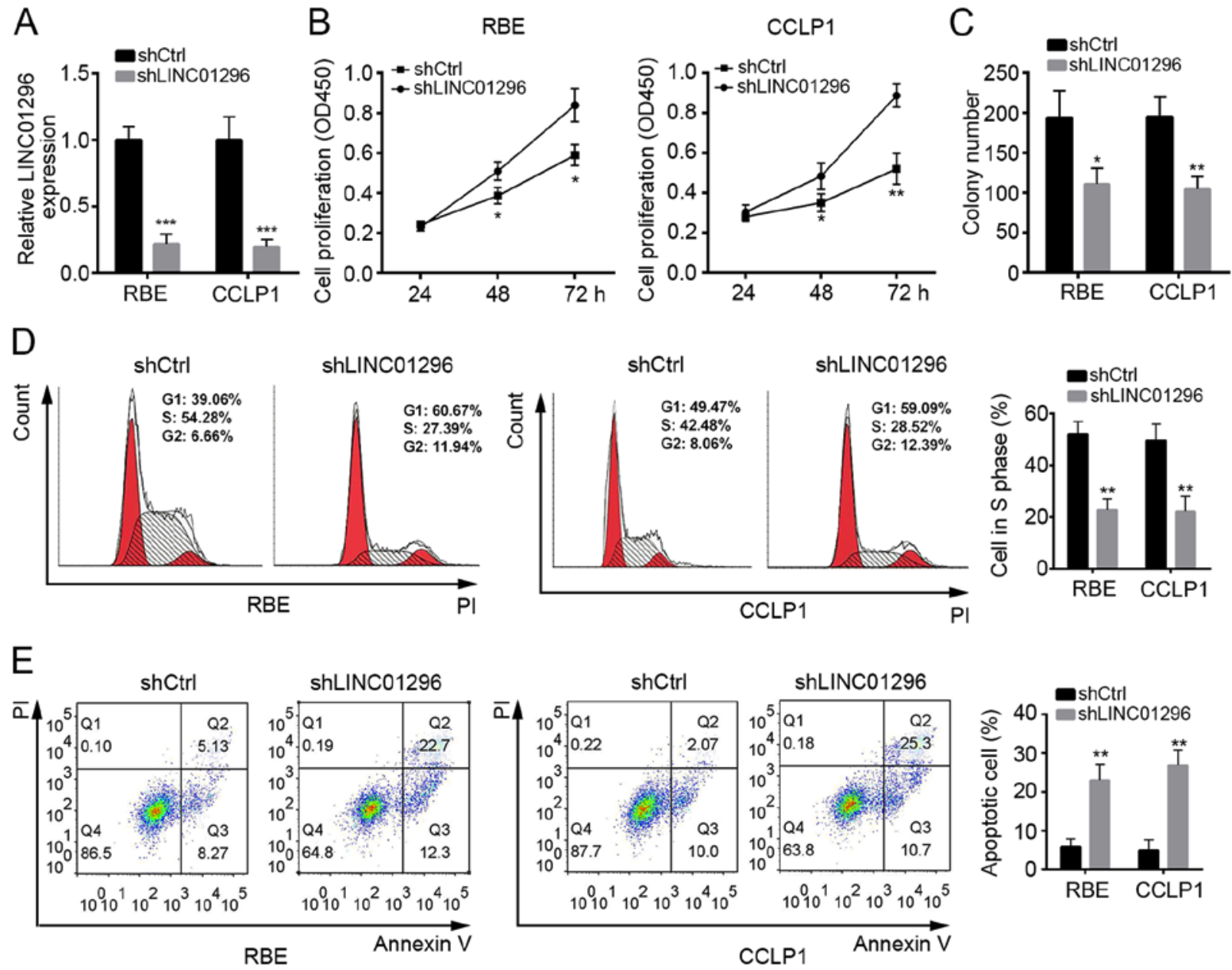

Figure 2. LINC01296 knockdown suppresses cell proliferation and promotes cell apoptosis. (A) RBE and CCLP1 cells were transfected with shLINC01296, followed by reverse transcription-quantitative polymerase chain reaction detection of LINC01296 expression. Cell proliferation was determined in (B) RBE and (C) CCLP1 cells transfected with shCtrl or shLINC01296 by CCK8 assay and colony formation assay. (D) Cell cycle distribution was determined by flow cytometry. (E) Cell apoptosis was measured in RBE and CCLP1 cells transfected with shCtrl or shLINC01296 by staining with Annexin V/PI. Data are presented as the mean \pm standard deviation. ${ }^{*} \mathrm{P}<0.05,{ }^{* * *} \mathrm{P}<0.01$ and ${ }^{* * *} \mathrm{P}<0.001$ vs. shCtrl. LINC01296, long intergenic non-protein coding RNA $1296 ;$ sh, short hairpin RNA; Ctrl, control; PI, propidium iodide.

positively associated with advanced clinical stages and lymph node metastasis. Furthermore, patients with higher expression of LINC01296 had a lower survival rate (Fig. 1F).

LINC01296 knockdown suppresses cell proliferation and promotes cell apoptosis. LINC01296 expression was highest in RBE and CCLP1 cells among the CCA cell lines (Fig. 1E). Thus, these two cell lines were selected for use in subsequent experiments. To explore whether LINC01296 influences the proliferation of RBE and CCLP1 cells, LINC01296 was knocked down by transfection with shLINC01296 plasmid (Fig. 2A). CCK 8 and colony formation assays demonstrated that LINC01296 knockdown significantly suppressed the viability and proliferation of RBE and CCLP1 cells (Fig. 2B and C). To further investigate the mechanism through which LINC01296 inhibited cell proliferation, the effects of LINC01296 on cell cycle distribution were determined. Flow cytometry analysis demonstrated that the percentage of cells in $\mathrm{S}$ phase was decreased in RBE and CCLP1 cells transfected with shLINC01296 compared with control shRNA (Fig. 2D), which suggested that LINC01296 promoted cell proliferation by regulating the cell cycle. Furthermore, Annexin V/PI staining demonstrated that LINC01296 knockdown significantly promoted the apoptosis of RBE and CCLP1 cells (Fig. 2E).

LINC01296 knockdown inhibits the migration and invasion of RBE and CCLP1 cells. To further define the effects of LINC01296 on tumor metastasis, wound healing assay and Transwell invasion assay were performed. LINC01296 knockdown significantly inhibited cell motility (Fig. 3A) and suppressed cell invasion (Fig. 3B) compared with control shRNA. These data suggested that LINC01296 inhibited metastatic capacity of RBE and CCLP1 cells.

LINC01296 interacts with miR-5095 in RBE and CCLPI cells. To explore the molecular mechanism by which LINC01296 inhibit CCA progression, the target miRNAs of LINC01296 were predicted with computational algorithms, including TargetScan (targetscan.org/vert_71/) and miRanda (34.236.212.39/microrna/getGeneForm.do). Among all candidates, miR-5095 ranked top. The predicted miR-5095 interaction site in LINC01296 is illustrated in Fig. 4A. RT-qPCR demonstrated that overexpression of LINC01296 reduced the level of miR-5095, while the miR-5095 mimic reduced LINC01296 


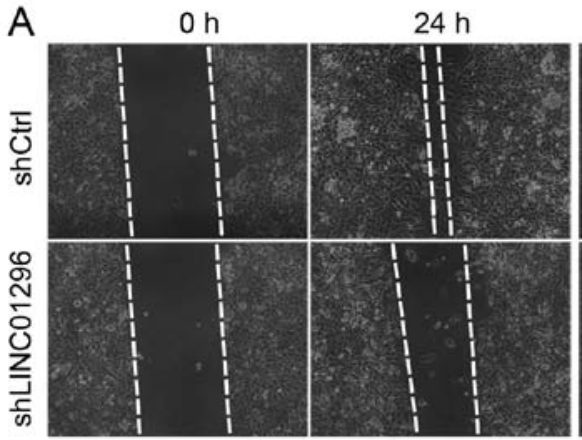

RBE

B

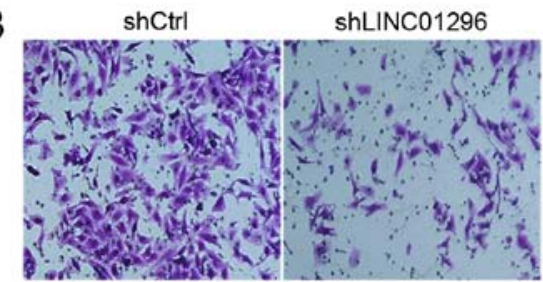

RBE
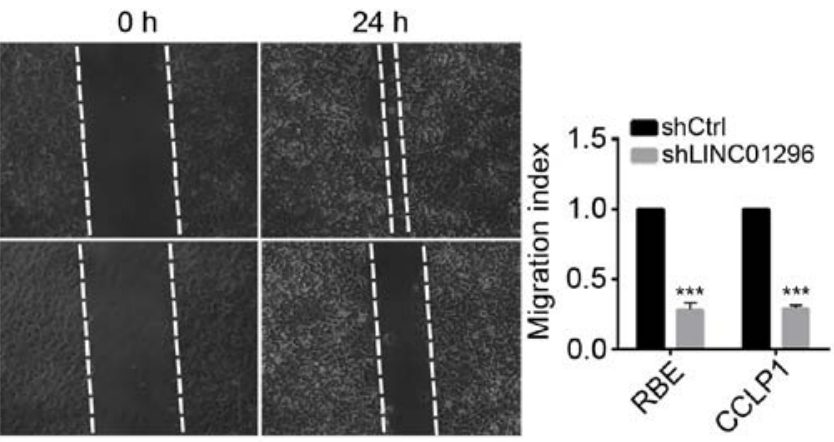

CCLP1

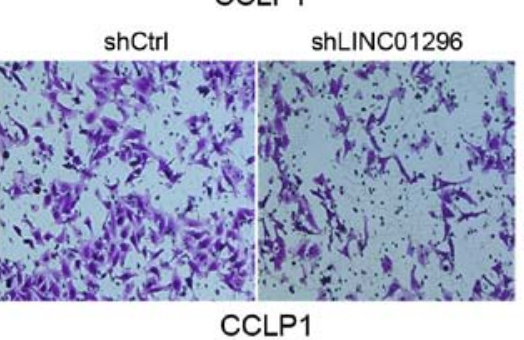

Figure 3. LINC01296 knockdown inhibits the migration and invasion of RBE and CCLP1 cells. (A) Cell migration was determined by wound healing assay in RBE and CCLP1 cells transfected with shCtrl or shLINC01296. (B) Cell invasion was determined by Transwell invasion assay in RBE and CCLP1 cells transfected with shCtrl or shLINC01296. Data are presented as the mean \pm standard deviation. ${ }^{* * *} \mathrm{P}<0.01$ and ${ }^{* * * *} \mathrm{P}<0.001$ vs. shCtrl. LINC01296, long intergenic non-protein coding RNA 1296; sh, short hairpin RNA; Ctrl, control.

A

LINC01296-WT: 5'---CAUGCCUGUAAUC---3'

miR-5095: 3'----GUGCGGACAUU---5'

LINC01296-Mut: 5'---CAUGGGACAUAUC---3'
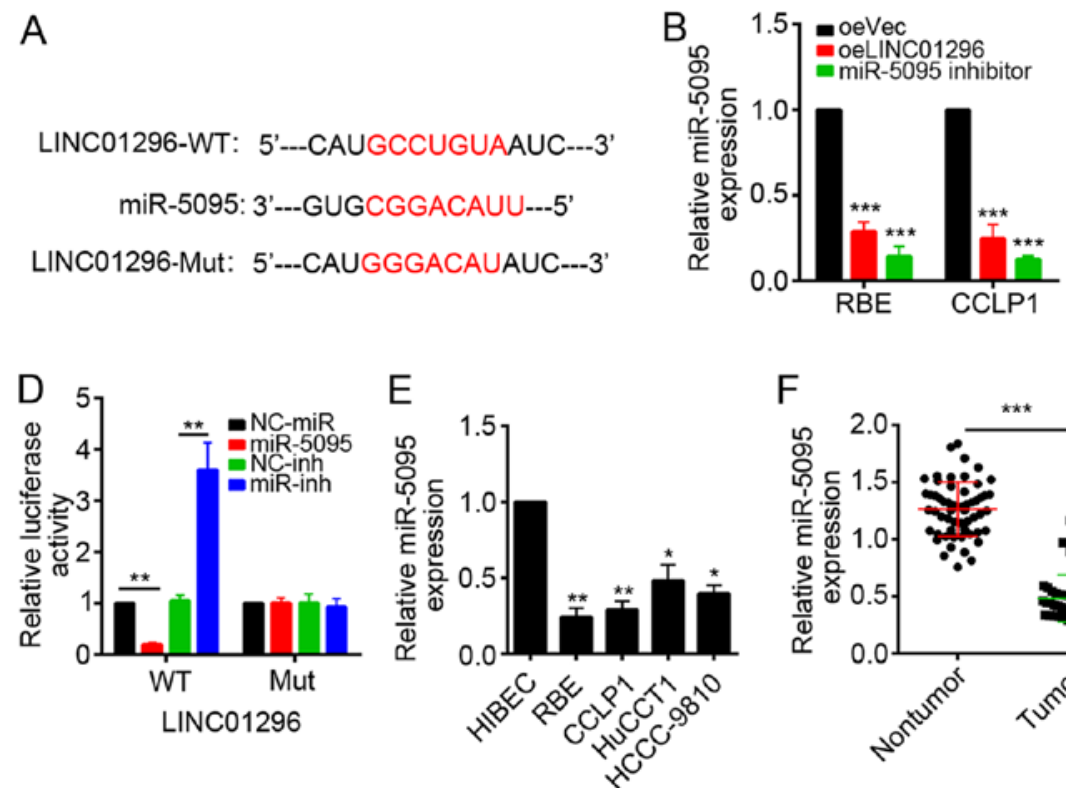

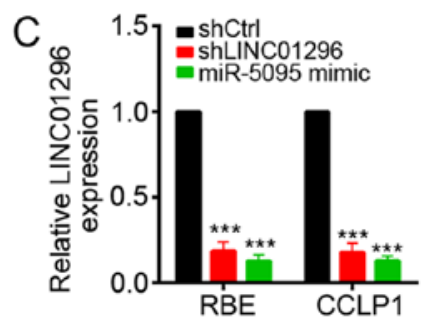

G

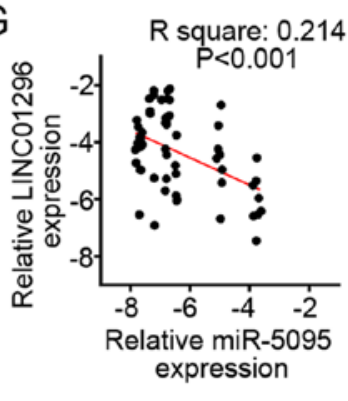

Figure 4. LINC01296 interacts with miR-5095 in RBE and CCLP1 cells. (A) miR-5095 binding sites in LINC01296 predicted by bioinformatics analysis. (B) Analysis of miR-5095 expression levels in RBE and CCLP1 cells transfected with LINC01296 overexpressing plasmid or miR-5095 inhibitor by RT-qPCR (C) Analysis of LINC01296 expression levels in RBE and CCLP1 cells transfected with shLINC01296 or miR-5095 mimic by RT-qPCR. ${ }^{* * *} \mathrm{P}<0.001 \mathrm{vs.} \mathrm{oeVec}$. (D) Luciferase reporter assays were performed using RBE cells co-transfected with the miR-5095 mimic or inhibitor and LINC01296-wt or LINC01296-mut reporter plasmid. ${ }^{* *} \mathrm{P}<0.01$. (E) The expression levels of miR-5095 were determined in CCA cell lines (RBE, CCLP1, HuCCT1 and HCCC-9810) and noncancerous cholangiocyte cell line (HIBEC) by RT-qPCR. ${ }^{*} \mathrm{P}<0.05$ and ${ }^{* * *} \mathrm{P}<0.01$ vs. HIBEC. (F) The expression of miR-5095 in CCA samples and nontumor tissues was determined by RT-qPCR. ${ }^{* * *} \mathrm{P}<0.001$. (G) Spearman's correlation analysis was used to determine the correlations between the levels of LINC01296 and miR-5095 in human CCA ( $\mathrm{n}=57)$. Data are presented as the mean \pm standard deviation. RT-qPCR, reverse transcription-quantitative polymerase chain reaction; CCA, cholangiocarcinoma; LINC01296, long intergenic non-protein coding RNA 1296; WT, wild-type; Mut, mutated; miR, microRNA; oeVec, overexpression empty vector; oeLINC01296, LINC01296 overexpression vector; sh, short hairpin RNA; Ctrl, control; NC, negative control; inh, miR inhibitor.

levels in RBE and CCLP1 cells (Fig. 4B and C). To further validate the interaction between LINC01296 and miR-5095, a dual-luciferase reporter assay was performed. Overexpression of miR-5095 significantly decreased the luciferase activity of the reporter containing wild-type LINC01296 in RBE cells, while it failed to repress the reported with mutated miR-5095 binding site (Fig. 4D). miR-5095 inhibitor also produced similar results (Fig. 4D). Furthermore, RT-qPCR 
A

$$
\begin{aligned}
& \text { MYCN-WT:5'---ACUGCCUGUAUA---3' } \\
& \text { miR-5095:3'----GUGCGGACAUU----5' }
\end{aligned}
$$

MYCN-Mut:5'---ACUGGGACAAUA---3'

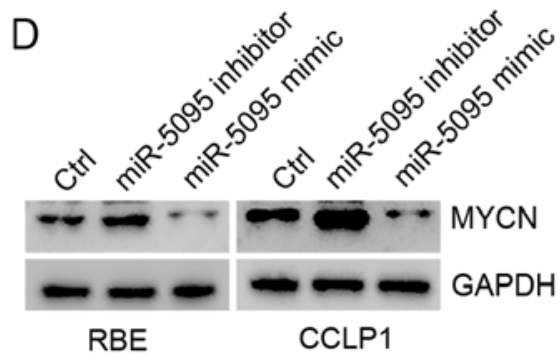

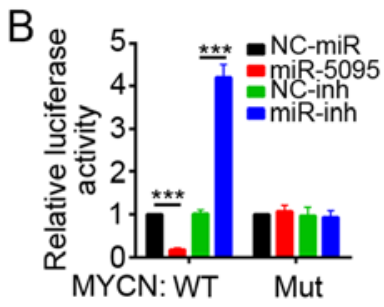

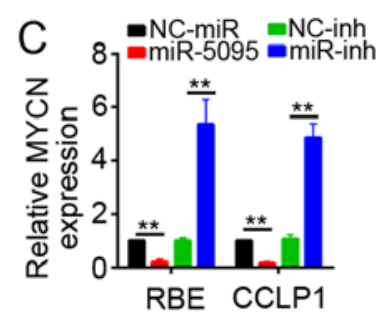

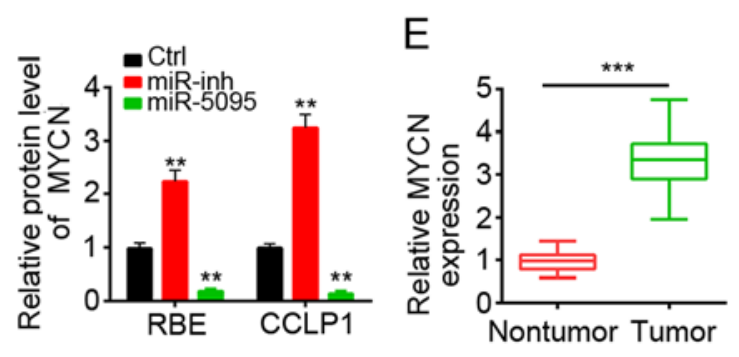

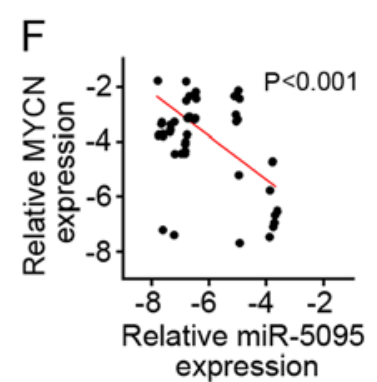

Figure 5. MYCN is a target of miR-5095. (A) Conservation of the miR-5095-targeting sites in the MYCN-3'UTR and its mutant sequence that abrogates MYCN binding to target mRNA. (B) Luciferase reporter assays were performed using RBE cells co-transfected with the miR-5095 mimic or inhibitor and MYCN-WT3'UTR or MYCN-Mut-3'UTR reporter plasmid. (C) mRNA and (D) protein levels of MYCN in RBE and CCLP1 cells transfected with the miR-5095 mimic or inhibitor were determined by RT-qPCR. (E) Expression of MYCN in cholangiocarcinoma samples and nontumor tissues was determined by RT-qPCR. (F) Spearman's correlation analysis was used to determine the correlations between the levels of MYCN and miR-5095 in human CCA (n=57). Data are presented as the mean \pm standard deviation. ${ }^{* *} \mathrm{P}<0.01$ and ${ }^{* * *} \mathrm{P}<0.001$. RT-qPCR, reverse transcription-quantitative polymerase chain reaction; MYCN, MYCN proto-oncogene bHLH transcription factor; WT, wild-type; miR, microRNA; Mut, mutated; NC, negative control; inh, miR inhibitor; Ctrl, control.

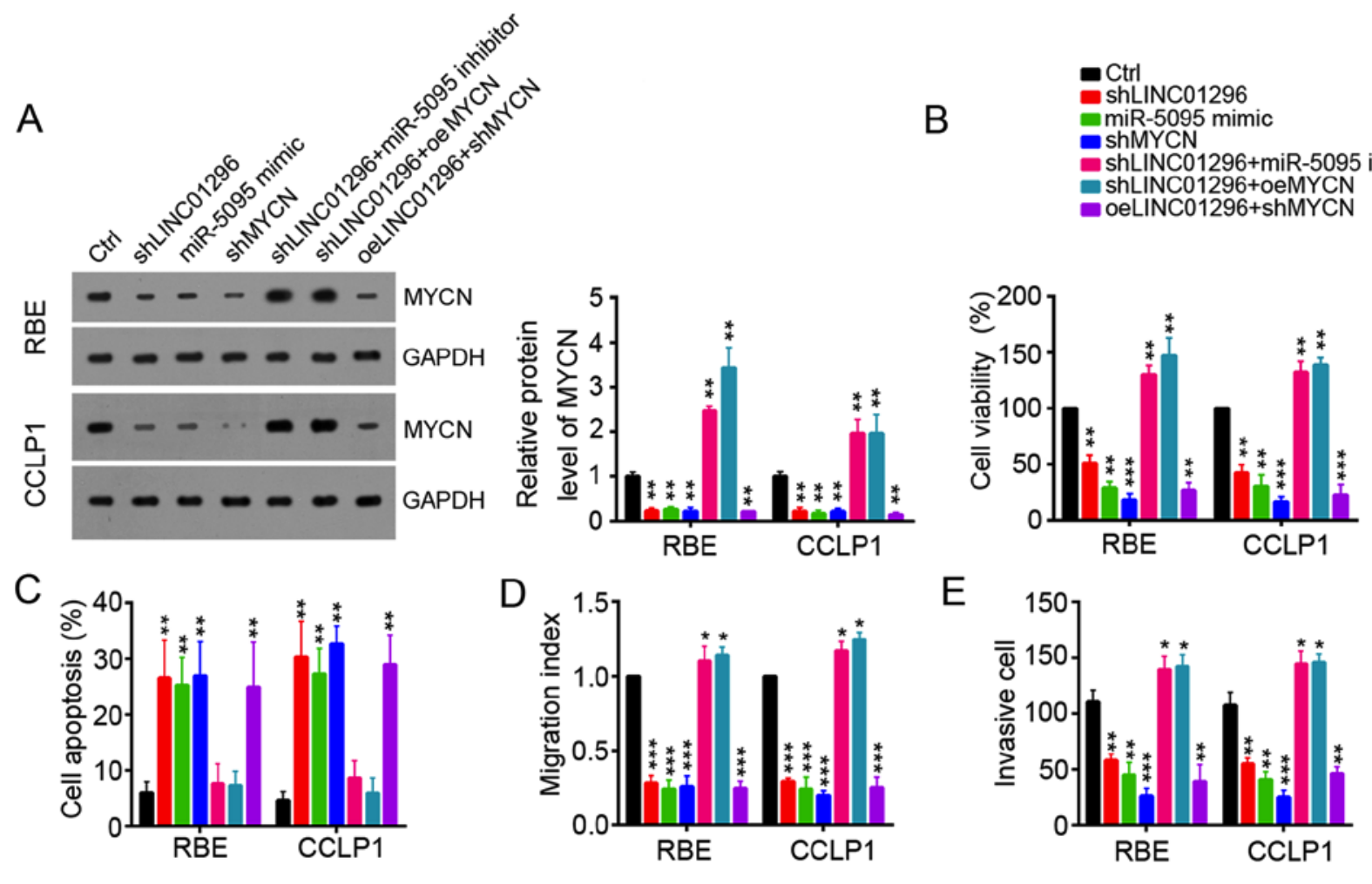

Figure 6. Restoration of MYCN reverses LINC01296 knockdown-induced inhibition of cell proliferation, migration, and invasion of RBE and CCLP1 cells (A) Knockdown of LINC01296 or miR-5095 inhibited the level of MYCN protein in RBE and CCLP1 cells as demonstrated by western blot analysis. (B) Cell viability, (C) apoptosis, (D) migration and (E) invasion were determined in RBE and CCLP1 cells transfected with shLINC01296, miR-5095 mimic, shMYCN, miR-5095 inhibitor or MYCN-overexpressing plasmid by CCK8, flow cytometry, wound-healing, and invasion assays, respectively. Data are presented as the mean \pm standard deviation. ${ }^{*} \mathrm{P}<0.05,{ }^{* *} \mathrm{P}<0.01$ and ${ }^{* * *} \mathrm{P}<0.001$ vs. Ctrl. Ctrl, control; sh, short hairpin RNA; LINC01296, long intergenic non-protein coding RNA 1296; miR, microRNA; MYCN, MYCN proto-oncogene bHLH transcription factor; oe, overexpression vector.

demonstrated that miR-5095 expression was lower in CCA cell lines than in HIBEC cells (Fig. 4E), and the expression of miR-5095 was lower in CCA tissues compared with nontumor tissues (Fig. 4F). Finally, in CCA tissues, miR-5095 expression was also inversely correlated with LINC01296 expression $(\mathrm{P}<0.001$, Spearman's rank test) (Fig. 4G). 
A

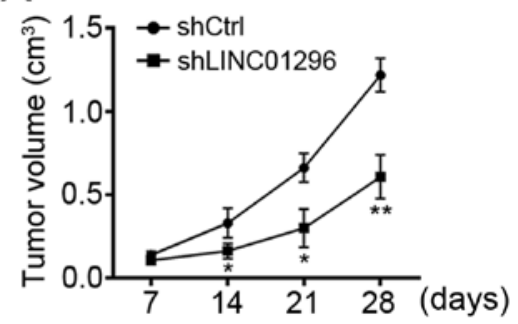

$\mathrm{B}$

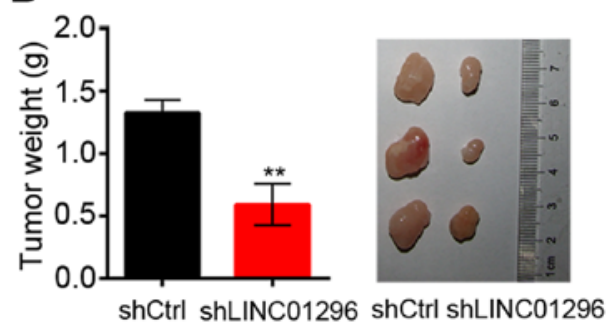

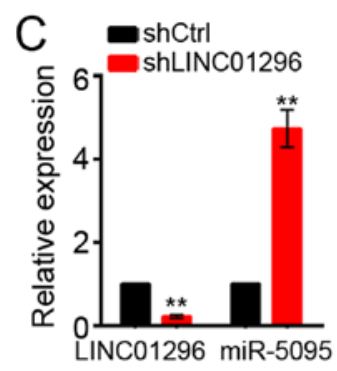

D
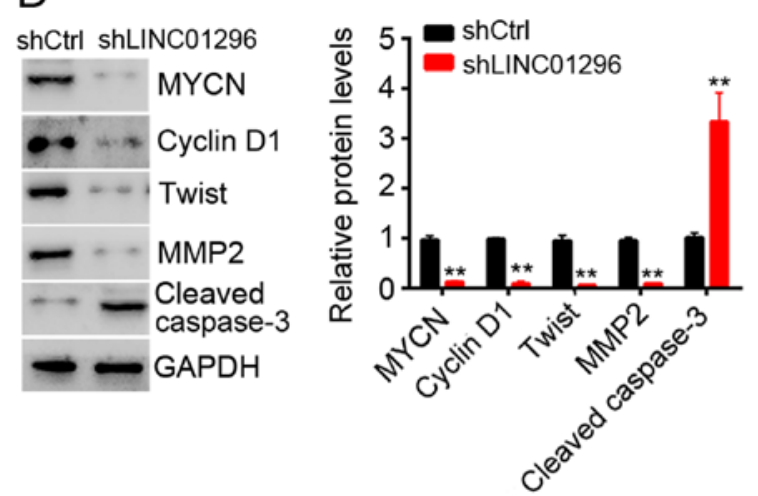

Figure 7. LINC01296 promoted tumor growth in vivo by activation of MYCN. (A) Tumor growth curves were established by measuring tumor volume every 7 for 28 days after injection. (B) Tumor weights isolated from nude mice in each treatment group were determined on day 28 after injection. (C) Relative LINC01296 and miR-5095 expression in xenograft tumors was determined by reverse transcription-quantitative polymerase chain reaction. (D) MYCN protein expression in xenograft tumors was determined by western blot. GAPDH was served as a loading control. Data are presented as the mean \pm standard deviation ${ }^{*} \mathrm{P}<0.05$ and ${ }^{* *} \mathrm{P}<0.01$ vs. shCtrl. sh, short hairpin RNA; Ctrl, control; LINC01296, long intergenic non-protein coding RNA 1296; miR, microRNA; MYCN proto-oncogene bHLH transcription factor; MMP2, matrix metalloproteinase-2.

MYCN is a target of miR-5095. To further explore the downstream target genes of miR-5095, prediction of miR-5095 targets was performed with computational algorithms, including TargetScan and miRanda. MYCN was selected. MYCN has been proven to promote tumor initiation and metastasis in various cancer types (26-28). The predicted miR-5095 interaction site in the MYCN 3'UTR is illustrated in Fig. 5A. To further validate the interaction between miR-5095 and MYCN, dual-luciferase reporter assay was performed. miR-5095 mimic significantly decreased the luciferase activity of the wild-type MYCN 3'UTR in RBE cells, while it failed to repress luciferase activity of the reported with mutated MYCN 3'UTR, and the miR-5095 inhibitor had the opposing effect (Fig. 5B). RT-qPCR and western blot analysis demonstrated that miR-5095 mimic suppressed the expression of MYCN in RBE and CCLP1 cells, and the miR-5095 inhibitor had the opposing effect (Fig. 5C and D). Furthermore, the expression of MYCN was upregulated in CCA samples compared with nontumor tissues (Fig. 5E). Additionally, in CCA tissues, miR-5095 expression was inversely correlated with MYCN expression $(\mathrm{P}<0.001$, Spearman's rank test) (Fig. 5F).

Restoration of MYCN reverses LINC01296 knockdowninduced inhibition of cell viability, migration, and invasion of RBE and CCLPI cells. To investigate whether the regulation of cell proliferation, migration and invasion of RBE and CCLP1 cells by LINC01296 is MYCN-dependent, knockdown of LINC01296, miR-5095, MYCN and/or overexpression of
MYCN were performed (Fig. 6A). Cell viability, apoptosis, migration and invasion were analyzed using CCK8, flow cytometry and Transwell assays, respectively. Knockdown of LINC01296 or MYCN and overexpression of miR-5095 suppressed cell viability, migration and invasion in RBE and CCLP1 cells, while miR-5095 inhibition or overexpression of MYCN reversed LINC01296 knockdown-induced inhibition of cell viability, migration and invasion (Fig. 6B-E).

LINC01296 promoted tumor growth in vivo by activation of MYCN. The biological effects of LINC01296 on CCA development were evaluated in a xenograft mouse model. RBE cells transfected with shCtrl or shLINC01296 were implanted subcutaneously into nude mice. Then tumor growth was evaluated every 7 days. LINC01296 knockdown significantly delayed tumor growth in vivo (Fig. 7A). At 5 weeks postimplantation, the nude mice were sacrificed, and tumors were harvested and weighed. LINC01296 knockdown significantly decreased the tumor size and weight (Fig. 7B). Subsequently, the expression levels of LINC01296, miR-5095 and MYCN were determined in tumor tissues by RT-qPCR and western blot analysis, respectively. Tumors derived from shLINC01296 cells exhibited a significant decrease in LINC01296 and MYCN expression, and an increase in miR-5095 expression (Fig. 7C and D). Furthermore, LINC01296 knockdown significantly inhibited the expression of proteins associated with tumor cell proliferation, migration and invasion, and increased the expression of caspase-3, which is crucial for cell apoptosis (Fig. 7D). 


\section{Discussion}

In recent decades, various diagnostic advances and therapeutic strategies have been used to treat CCA. However, the overall survival rates of patients with CCA remain low due to tumor recurrence and metastasis (17). Thus, in order to develop novel and effective therapeutic approaches for CCA treatment, there is an urgent need to fully understand the molecular mechanisms that regulate CCA initiation and progression.

Recent evidence has indicated that $97 \%$ transcripts produced from the human genome are short or lncRNAs with no, or limited, protein-coding potential (29). The role of lncRNAs in human cancer has been a developing field in the recent years. A large number of studies demonstrated that IncRNAs are closely associated with the development and progression of various tumor types, including CCA (16). For instance, the lncRNA HOX transcript antisense RNA activates the Hippo pathway in renal cell carcinoma (30). IncRNA HOXA cluster antisense RNA 3 promotes tumor progression and predicts poor prognosis in glioma (31). However, the functions of the majority of lncRNAs in CCA remain elusive. In the current study, LINC01296 was significantly upregulated in CCA. A previous study reported that LINC01296 is associated with poor prognosis in prostate cancer and promotes cancer cell proliferation and metastasis (32). LINC01296 was also demonstrated to promote colorectal cancer and bladder cancer progression in vitro $(33,34)$. However, the functions of LINC01296 in CCA have not been defined. In the current study, LINC01296 knockdown significantly inhibited cell proliferation, migration and invasion, and led to increased cell apoptosis in CCA. These findings suggested that LINC01296 may have an oncogenic role in CCA progression.

A previous study reported that IncRNAs can exert biological functions via a variety of mechanisms, including transcriptional and post-transcriptional regulation. For example, lymphocyte-specific protein 1 pseudogene recruited DNA-binding protein SATB1 to initiate transcription of hes family bHLH transcription factor 6 and promote hepatocellular carcinoma progression (13). Additionally, lncRNA MALAT1 acted as a competing endogenous RNA of miR-144-3p to promote metastasis and proliferation in osteosarcoma cells (19). Zhu et al (22) reported that lnc- $\beta$-Catm interacts with $\beta$-catenin to prevent it degradation and then sustains liver cancer stem cell self-renewal. In the current study, LINC01296 was predicted to interact with miR-5095 by computational algorithms. A luciferase activity reporter assay then validated that LINC01296 sponged miR-5095 in CCA cells. Additionally, expression of LINC01296 was negatively correlated with miR-5095 in CCA. miRNAs can target the 3'UTR of mRNAs for degradation and then regulate gene expression. The functions of miR-5095 were unknown prior to the current study. Computational algorithms predicted that $\mathrm{MYCN}$, an oncogene in various types of human cancer $(28,35,36)$, was a potential target of miR-5095. Luciferase activity assay, RT-qPCR and western blot, demonstrated that miR-5095 bound to the 3'UTR of MYCN and downregulated its expression in CCA. Finally, restoration of MYCN expression in RBE and CCLP1 cells rescued the LINC01296 knockdown-induced inhibition of cell viability, migration and invasion. Therefore, our findings indicated that LINC01296 promoted cell proliferation, migration and invasion by sponging miR-5095 to upregulate MYCN expression.

In summary, the results of the current study demonstrated that LINC01296 was significantly upregulated in CCA tumors and cell lines compared with normal tissue and cells, and LINC01296 was associated with cell growth, migration and invasion in vitro and in vivo. The present study revealed a novel mechanism of a LINC01296/miR-5095/MYCN axis that regulates CCA development and progression. The study demonstrated that LINC01296 may act as a novel diagnostic and prognostic indicator, and a therapeutic target for CCA treatment.

\section{Acknowledgements}

Not applicable.

\section{Funding}

This study was supported by the National Natural Science Foundation of Guangdong (grant nos. 2014A030310160, 2014A030310015 and 2015A030313466), the Science and Technology Planning Project of Guangdong (grant nos. 2016A02021521 and 2016A020215038), the Science and Technology Program of Guangzhou (grant nos. 201707010469 and 201607010033), and the National Natural Science Foundation Project (grant nos. 81602109 and 81602331).

\section{Availability of data and materials}

The datasets used and/or analyzed during the current study are available from the corresponding author on reasonable request.

\section{Authors' contributions}

DZ and HL designed and performed the experiments; JX, DJ, LC and XJ contributed to the data analysis; XY enrolled patients and measured the RNA levels in the clinical samples; PX initiated the work and wrote the manuscript; and all authors have read and approved the final version of the manuscript.

\section{Ethics approval and consent to participate}

The use of human tissues was approved by the Ethics Committee of the Second Affiliated Hospital of Guangzhou Medical University and patient consent was obtained.

\section{Consent for publication}

Not applicable.

\section{Competing interests}

The authors declare that they have no competing interests.

\section{References}

1. Razumilava N and Gores GJ: Cholangiocarcinoma. Lancet 383: 2168-2179, 2014.

2. Shaib YH, Davila JA, McGlynn K and El-Serag HB: Rising incidence of intrahepatic cholangiocarcinoma in the United States: A true increase? J Hepatol 40: 472-477, 2004. 
3. Welzel TM, Graubard BI, El-Serag HB, Shaib YH, Hsing AW, Davila JA and McGlynn KA: Risk factors for intrahepatic and extrahepatic cholangiocarcinoma in the United States: A population-based case-control study. Clin Gastroenterol Hepatol 5 : 1221-1228, 2007.

4. Xiong J, Wang Y, Huang H, Bian J, Wang A, Long J, Zheng Y, Sang $\mathrm{X}, \mathrm{Xu} \mathrm{Y}, \mathrm{Lu} \mathrm{X}$, et al: Systematic review and metaanalysis: Cholecystectomy and the risk of cholangiocarcinoma. Oncotarget 8: 59648-59657, 2017.

5. Liu S, Jiang B, Li H, He Z, Lv P, Peng C, Wang Y, Cheng W, $\mathrm{Xu} \mathrm{Z}$, Chen W, et al: Wipl is associated with tumorigenity and metastasis through MMP-2 in human intrahepatic cholangiocarcinoma. Oncotarget 8: 56672-56683, 2017.

6. Dover LL, Jacob R, Wang TN, Richardson JH, Redden DT, Li P and DuBay DA: Improved postoperative survival for intraductalgrowth subtype of intrahepatic cholangiocarcinoma. Am Surg 82: 1133-1139, 2016

7. Smith-Cohn MA, Gill D, Voorhies BN, Agarwal N and GarridoLaguna I: Case report: Pembrolizumab-induced type 1 diabetes in a patient with metastatic cholangiocarcinoma. Immunotherapy 9 : 797-804, 2017.

8. Yang J, Liu Y, Wang B, Lan H, Liu Y, Chen F, Zhang J and Luo J: Sumoylation in p27kip1 via RanBP2 promotes cancer cell growth in cholangiocarcinoma cell line QBC939. BMC Mol Biol 18: 23, 2017.

9. Loosen SH, Roderburg C, Kauertz KL, Pombeiro I, Leyh C, Benz F, Vucur M, Longerich T, Koch A, Braunschweig T et al: Elevated levels of circulating osteopontin are associated with a poor survival after resection of cholangiocarcinoma. J Hepatol 67: 749-757, 2017.

10. Svoboda P: Long and small noncoding RNAs during oocyte-toembryo transition in mammals. Biochem Soc Trans 45: 1117-1124, 2017.

11. Liu B, Ye B, Yang L, Zhu X, Huang G, Zhu P, Du Y, Wu J, Qin X, Chen $\mathrm{R}$, et al: Long noncoding RNA $\operatorname{lncKdm} 2 \mathrm{~b}$ is required for ILC3 maintenance by initiation of Zfp292 expression. Nat Immunol 18: 499-508, 2017.

12. Zhu P, Wang Y, Wu J, Huang G, Liu B, Ye B, Du Y, Gao G, Tian Y, He L, et al: LncBRM initiates YAP1 signalling activation to drive self-renewal of liver cancer stem cells. Nat Commun 7 : 13608,2016

13. Xu YC, Liang CJ, Zhang DX, Li GQ, Gao X, Fu JZ, Xia F, Ji JJ, Zhang LJ, Li GM, et al: LncSHRG promotes hepatocellular carcinoma progression by activatingHES6. Oncotarget 8: 70630-70641, 2017.

14. Monteleone NJ and Lutz CS: miR-708-5p: A microRNA with emerging roles in cancer. Oncotarget 8: 71292-71316, 2017.

15. Faghihi $\mathrm{F}$ and Moustafa AA: Impaired neurogenesis of the dentate gyrus is associated with pattern separation deficits: A computational study. J Integr Neurosci 15: 277-293, 2016.

16. Xu Y, Leng K, Li Z, Zhang F, Zhong X, Kang P, Jiang X and Cui Y: The prognostic potential and carcinogenesis of long noncoding RNA TUG1 in human cholangiocarcinoma. Oncotarget 8 : 65823-65835, 2017.

17. Shi X, Zhang H, Wang M, Xu X, Zhao Y,He R, Zhang M, Zhou M, Li X, Peng F, et al: LncRNA AFAP1-AS1 promotes growth and metastasis of cholangiocarcinoma cells. Oncotarget 8: 58394-58404, 2017

18. Li J, Zhang Q, Fan X, Mo W, Dai W, Feng J, Wu L, Liu T, Li S, Xu S, et al: The long noncoding RNA TUG1 acts as a competing endogenous RNA to regulate the Hedgehog pathway by targeting miR-132 in hepatocellular carcinoma. Oncotarget 8 : 65932-65945, 2017.

19. Wang Y, Zhang Y, Yang T, Zhao W, Wang N, Li P, Zeng X and Zhang W: Long non-coding RNA MALAT1 for promoting metastasis and proliferation by acting as a ceRNA of miR-144-3p in osteosarcoma cells. Oncotarget 8: 59417-59434, 2017.

20. Puik JR, Meijer LL, Le Large TYS, Prado MM, Frampton AE, Kazemier $\mathrm{G}$ and Giovannetti E: miRNA profiling for diagnosis, prognosis and stratification of cancer treatment in cholangiocarcinoma. Pharmacogenomics 18: 1343-1358, 2017.

21. Wang Q, Tang H, Yin S and Dong C: Downregulation of microRNA-138 enhances the proliferation, migration and invasion of cholangiocarcinoma cells through the upregulation of RhoC/p-ERK/MMP-2/MMP-9. Oncol Rep 29: 2046-2052, 2013.
22. Zhu P, Wang Y, Huang G, Ye B, Liu B, Wu J, Du Y, He L and Fan Z: lnc- $\beta$-Catm elicits EZH2-dependent $\beta$-catenin stabilization and sustains liver CSC self-renewal. Nat Struct Mol Biol 23: 631-639, 2016.

23. Chen ZZ, Huang L, Wu YH, Zhai WJ, Zhu PP and Gao YF: LncSox 4 promotes the self-renewal of liver tumour-initiating cells through Stat3-mediated Sox4 expression. Nat Commun 7: 12598, 2016.

24. Wang P and Lv L: miR-26a induced the suppression of tumor growth of cholangiocarcinoma via KRT19 approach. Oncotarget 7: 81367-81376, 2016.

25. Zhao L, Zhang Y and Zhang Y: Long noncoding RNA CASC2 regulates hepatocellular carcinoma cell oncogenesis through miR-362-5p/Nf-кB axis. J Cell Physiol: Jan 10, 2018 (Epub ahead of print). doi: 10.1002/jep.26446.

26. Zhu S, Zhang X, Weichert-Leahey N, Dong Z, Zhang C, Lopez G, Tao T, He S, Wood AC, Oldridge D, et al: LMO1 synergizes with MYCN to promote neuroblastoma initiation and metastasis. Cancer Cell 32: 310-323.e5, 2017.

27. Cao Y, Jin Y, Yu J, Wang J, Qiu Y, Duan X, Ye Y, Cheng Y, Dong L, Feng X, et al: Clinical evaluation of integrated panel testing by next-generation sequencing for somatic mutations in neuroblastomas with MYCN unamplification. Oncotarget 8: 49689-49701, 2017.

28. Ambrosio S, Amente S, Saccà CD, Capasso M, Calogero RA, Lania L and Majello B: LSD1 mediates MYCN control of epithelial-mesenchymal transition through silencing of metastatic suppressor NDRG1 gene. Oncotarget 8: 3854-3869, 2017.

29. Zhang JY, Weng MZ, Song FB, Xu YG, Liu Q, Wu JY, Qin J, Jin T and Xu JM: Long noncoding RNA AFAP1-AS1 indicates a poor prognosis of hepatocellular carcinoma and promotes cell proliferation and invasion via upregulation of the RhoA/Rac2 signaling. Int J Oncol 48: 1590-1598, 2016.

30. Hu G, Dong B, Zhang J, Zhai W, Xie T, Huang B, Huang C, Yao X, Zheng J, Che J, et al: The long noncoding RNA HOTAIR activates the Hippo pathway by directly binding to SAV1 in renal cell carcinoma. Oncotarget 8: 58654-58667, 2017.

31. Wu F, Zhang C, Cai J, Yang F, Liang T, Yan X, Wang H, Wang $\mathrm{W}$, Chen $\mathrm{J}$ and Jiang T: Upregulation of long noncoding RNA HOXA-AS3 promotes tumor progression and predicts poor prognosis in glioma. Oncotarget 8: 53110-53123, 2017.

32. Wu J, Cheng G, Zhang C, Zheng Y, Xu H, Yang H and Hua L: Long noncoding RNA LINC01296 is associated with poor prognosis in prostate cancer and promotes cancer-cell proliferation and metastasis. Onco Targets Ther 10: 1843-1852, 2017.

33. Yuan Z, Yu X, Ni B, Chen D, Yang Z, Huang J, Wang J, Chen D and Wang L: Overexpression of long non-coding RNA-CTD903 inhibits colorectal cancer invasion and migration by repressing $\mathrm{Wnt} / \beta$-catenin signaling and predicts favorable prognosis. Int J Oncol 48: 2675-2685, 2016.

34. Seitz AK, Christensen LL, Christensen E, Faarkrog K, Ostenfeld MS, Hedegaard J, Nordentoft I, Nielsen MM, Palmfeldt J, Thomson M, et al: Profiling of long non-coding RNAs identifies LINC00958 and LINC01296 as candidate oncogenes in bladder cancer. Sci Rep 7: 395, 2017.

35. Tsubota S, Kishida S, Shimamura T, Ohira M, Yamashita S, Cao D, Kiyonari S, Ushijima T and Kadomatsu K: PRC2-mediated transcriptomic alterations at the embryonic stage govern tumorigenesis and clinical outcome in MYCN-Driven neuroblastoma. Cancer Res 77: 5259-5271, 2017.

36. Sun G, Lu J, Zhang C, You R, Shi L, Jiang N, Nie D, Zhu J, Li M and Guo J: MiR-29b inhibits the growth of glioma via MYCN dependent way. Oncotarget 8: 45224-45233, 2017.

This work is licensed under a Creative Commons Attribution-NonCommercial-NoDerivatives 4.0 International (CC BY-NC-ND 4.0) License. 